\title{
Amido resistente, um potencial ingrediente para ser considerado em alimentos funcionais para cães
}

\author{
Reis, J.S. ${ }^{1}$; Ogoshi, R.C.S. ${ }^{2}$; França, J. ${ }^{3}$; Figueiredo, M.L. ${ }^{4}$; Borges, L.A. ${ }^{5}$ e Zangeronimo, M.G. ${ }^{1}$
}

'Departamento de Medicina Veterinária. Universidade Federal de Lavras. Lavras. Brasil.

${ }^{2}$ Mestrado Acadêmico. Universidade Alto Vale do Rio Peixe. Caçador. Brasil.

${ }^{3}$ Faculdade de Medicina Veterinária. Universidade Federal de Uberlândia. Uberlândia. Brasil.

${ }^{4}$ Centro Universitario de Brusque. Brusque. Brasil.

${ }^{5}$ Centro Universitário para o Desenvolvimento do Alto do Vale do Itajaí. Rio do Sul. Brasil.

\section{PaLAVRAS CHAVE ADICIONAIS}

Canino.

Índice glicêmico.

Qualidade fecal.

Saude intestinal.

\section{RESUMO}

Além da função nutricional, o amido desempenha importante papel no processamento do alimento extrusado para cães. Por outro lado, grandes modificações ocorreram nos últimos tempos no que concerne ao conhecimento do papel dos carboidratos na nutrição e saúde tanto de humanos quanto demais espécies animais. Durante muitos anos acreditou-se na completa digestão e absorção do amido no intestino delgado, no entanto, sabe-se atualmente que uma parcela do amido pode resistir à ação de enzimas digestivas sendo, portanto, denominado de amido resistente (AR). O AR pode provir tanto por interferência no processamento do amido, quanto de fontes comerciais obtidas por processo patenteado ou naturais, por exemplo, alguns vegetais que o contém em altas concentrações. Em diversas espécies de animais estudadas foram atribuídos vários benefícios fisiológicos e/ou metabólicos ao AR, tais como: a melhora na resposta glicêmica e insulinêmica; melhora no perfil lipídico; a redução no valor calórico dos alimentos; o aumento da saciedade e absorção de micronutrientes; além de agir como prebiótico considerado promotor da saúde do trato gastrintestinal. Entretanto, para a espécie canina poucos os trabalhos enfatizaram o tema. Deste modo, o foco principal desta revisão foi a compilação de estudos a fim de obter melhor entendimento dos efeitos biológicos diferenciados atribuídos ao AR e seu potencial como componente nas dietas para cães.

\section{Resistant starch, a potential ingredient to be considered in functional foods for dogs}

\begin{abstract}
SUMMARY
Besides the nutritional function the starch plays an important role in the processing of extruded dog food. On the other hand, major changes have occurred in recent times regarding the knowledge of the role of carbohydrates in the nutrition and health of both humans and other animal species. For many years believed in complete digestion and absorption of starch in the small intestine, however, it is now known that a portion of the starch can resist the action of digestive enzymes therefore called resistant starch (RS). The RS can come either from interference in the processing of starch or from commercial sources obtained by patented or natural process, for example, some vegetables that contain it in high concentrations. In many species of animals the physiological and/or metabolic benefits were attributed from RS, such as: improvement glycemic and insulinemic response; improvement in lipid profile; reduction in the calorific value of food; increased satiety and absorption of micronutrients; besides acting as a prebiotic considered a health promoter of the gastrointestinal tract. However, for the canine specie few studies have been emphasized this matter. Thus, the aim of this review was gather studies to obtain a better understanding of the differentiated biological effects attributed to RS and its potential as a component in diets for dogs.
\end{abstract}

\section{INTRODUÇÃO}

A nutrição de animais de companhia vem buscando aperfeiçoar o estado nutricional e de bem-estar com o intuito de aumentar a longevidade com qualidade de vida. Assim, as pesquisas nesse setor, igualmente as pesquisas da nutrição humana, estão direcionadas para obter alimentos nutricionalmente adequados com o uso de ingredientes que tragam benefícios à saúde.

O amido é um homopolissacarídeo amplamente distribuído em diversas espécies de vegetais como um carboidrato de reserva comum em grãos de cereais, raízes e tubérculos. Além da função nutricional, como 
fonte de glicose, o amido desempenha importante papel no processamento de alimentos, principalmente os extrusados. Sabe-se que a maioria dos alimentos caninos e felinos disponíveis comercialmente são fabricados com a tecnologia de cozimento por extrusão e os grãos, que possuem de 50 a 90\% de amido, são os ingredientes mais utilizados neste processamento (Crane et al., 2000) aonde chegam a compor até 50\% das dietas (Spears e Fahey Junior, 2004).

Por outro lado, nas últimas décadas começou a ser observado que uma fração do amido escapava à digestão no intestino delgado de indivíduos saudáveis, chegando ao cólon, onde servia como substrato para a microbiota intestinal. A esta fração deu-se o nome de amido resistente (AR). Ao longo dos anos, foram sendo observados que determinados efeitos fisiológicos, inicialmente atribuídos às fibras alimentares, também poderiam ser desempenhados pelo AR (Lobo e Silva, 2003). Pesquisas foram desenvolvidas em humanos e ratos com objetivo de quantificar o AR em diversos alimentos, assim como avaliar a inclusão deste na alimentação usual, culminando com a determinação de seus efeitos benéficos. Até então, os efeitos observados do AR incluem: melhora na resposta glicêmica, insulinêmica e no perfil lipídico; redução no valor calórico dos alimentos; aumento da saciedade e absorção de alguns micronutrientes; além de agir como prebiótico estimulando o crescimento e atividade de bactérias benéficas colônicas, mantendo, consequentemente, a saúde do trato gastrintestinal. Deste modo, o AR pode ser incluído na categoria dos alimentos funcionais que se refere aos gêneros alimentícios que podem proporcionar benefícios nutricionais, dietéticos e metabólicos específicos, contribuindo assim no controle e redução do risco de doenças. Contudo, Peixoto et al. (2017) afirma que poucos estudos têm abordado sobre o AR e sua administração para cães.

Nesse sentido, uma vez ocorreram grandes modificações no que concerne ao conhecimento do papel dos carboidratos na nutrição e saúde tanto de humanos quanto demais espécies animais, o presente artigo tem por objetivo reunir estudos sobre o AR visando obter um maior entendimento sobre seu potencial como componente nas dietas funcionais para cães.

\section{O AMIDO E SUA IMPORTÂNCIA NO PROCESSO DE EXTRUSÃO}

Estruturalmente, o amido é um polímero de glicose composto por cadeias de amilose e amilopectina. A amilose é formada por unidades de glicose unidas por ligações glicosídicas $\alpha-1,4$ originando uma cadeia linear, já a amilopectina é formada por unidades desse carboidrato unidas por ligações $\alpha-1,4$ e $\alpha-1,6$, formando uma estrutura ramificada (Walter et al., 2005). A proporção desses dois polissacarídeos varia de acordo com a origem botânica do amido (Teste et al., 2004). A maioria dos amidos contém 200 a $250 \mathrm{~g}$ de amilose $/ \mathrm{kg}$, entretanto, alguns cereais denominados cerosos (waxy), tais como o milho, o arroz e a cevada, contém muito pouca amilose $(<1 \%)$; e outros amidos tipo amylomaize podem conter cerca de $650 \mathrm{~g}$ de amilose/kg (Parker e Ring, 2001).

De acordo com a velocidade de digestão in vitro, o amido pode ser classificado como amido rapidamen- te ou lentamente digerível e como amido resistente (AR). O rapidamente digerível (ARD) é aquele que ao ser submetido à incubação com amilase pancreática e amiloglicosidase em uma temperatura de $37^{\circ} \mathrm{C}$, converte-se completamente em glicose em até 20 minutos (Englyst et al., 1982). Este consiste principalmente de amido amorfo e disperso, sendo encontrado em grande quantidade em alimentos ricos em amido cozido por calor úmido como pão e batatas. $\mathrm{O}$ amido lentamente digerido (ALD) também é completamente digerido, entretanto em até 120 minutos nas condições de incubação anteriores. Nesta categoria incluem o amido amorfo fisicamente inacessível e o amido cru (Sajilata et al., 2006). Já o AR, é aquele que resiste após 120 minutos de digestão in vitro (Englyst et al., 1992) e pode ser mensurado pela seguinte equação:

\section{$\mathrm{AR}=$ total de amido da amostra-(ARD-ALD)}

Além da função nutricional como fonte de glicose, o amido desempenha importante papel no processamento de alimentos, principalmente os extrusados. A tecnologia de cozimento por extrusão é caracterizada como um processo de alta temperatura e curto tempo (Dziezak, 1989) em que a mistura de alimento é exposta a uma alta pressão e temperatura $\left(80-200^{\circ} \mathrm{C}\right)$ por um período de tempo relativamente curto (10-270s.) (Lankhorst et al., 2007). Nesse processo, assim como em outros que envolvem calor e presença de água, ocorre a gelatinização do amido que é caracterizada pelo rompimento de grânulos amiláceos, com desestruturação da ordem molecular e mudanças irreversíveis em suas propriedades (Denardin e da Silva, 2009). De uma maneira geral, a extrusão e outros processos que resultam na gelatinização aumentam a digestibilidade de varias fontes de grãos cereais para os carnívoros (Spears e Fahey Junior, 2004). Existem muitos fatores que afetam a temperatura de gelatinização, sendo o principal deles a presença de água, uma vez que ela atua como agente plastificante nos cristais de amido. A sua presença diminuirá a temperatura de transição vítrea, diminuindo consequentemente, a temperatura de desorganização dos cristais (Denardin e da Silva, 2009) e aumentando a suscetibilidade do amido à digestão pelas amilases do trato gastrointestinal (Björck et al., 1994). Outros fatores que podem interferir na gelatinização incluem a estrutura molecular da amilopectina (comprimento de cadeia, extensão de ramificação, peso molecular), a composição do amido (proporção amilose:amilopectina e teor de fósforo) e a arquitetura granular (proporção de regiões cristalinas e amorfas) (Singh et al., 2003).

O processo de extrusão e a apresentação do alimento extrusado seco estão diretamente relacionados ao processo de gelatinização do amido, e o fato de alterar a proporção entre amilose e amilopectina favorece a obtenção de produtos com aparências diferenciadas. Segundo Huang (2001), um aumento no teor de amilose aumenta a crocância enquanto um aumento no teor de amilopectina melhora a expansão do produto extrusado. O grânulo de amilose, devido a sua estrutura em hélice, forma um filamento menor, mais fino e com menor viscosidade após sua gelatinização, ou seja, um excesso de amilose em relação à amilopectina dificulta a expansão do produto. Os grânulos de amilopectina, 
por apresentarem ramificações entre suas moléculas, formam, após a gelatinização, filamentos mais longos, com maior viscosidade e aderência sendo, portanto, realmente efetivos no processo de expansão. Entretanto, ao utilizar alto teor de amilopectina, pode diminuir a uniformidade do produto (Lara, 2005).

O amido gelatinizado quando é resfriado e armazenado pode sofrer um fenômeno denominado retrogradação, ou seja, transformação em amido retrogradado que corresponde a um dos tipos de AR, o tipo 3. Segundo Denardin e da Silva (2009) o fenômeno da retrogradação é complexo e varia de intensidade conforme diversos fatores, como: temperatura e tempo de armazenamento, $\mathrm{pH}$, fonte do amido, presença de outros componentes (lipídios, eletrólitos e açúcares) e condições de processamento. Quanto a este último, acredita-se que quando o amido é cozido em água acima de sua temperatura de gelatinização e na sequência é resfriado ocorre a reestruturação o do amido em um estado mais ordenado com áreas cristalinas e resistentes à hidrólise por amilases (Kleessen et al., 1997). Deste modo, torna-se tecnicamente possível aumentar o teor de amido retrogradado por meio da alteração das condições do processamento do alimento. No intestino grosso, o amido retrogradado pode ser fermentado ou excretado, exibindo de tal modo propriedades das fibras que são fermentáveis, entretanto, há alguns amidos retrogradados que são menos fermentáveis, resultando em propriedades laxativas em alguns animais (Tran et al., 2008)

\section{CARACTERIZAÇ̃̃O DO AMIDO RESISTENTE}

O termo AR foi inicialmente sugerido por Englyst et al. (1982) no desenvolvimento de um ensaio in vitro com polissacarídeos não amiláceos onde observaram que alguns amidos permaneceram após hidrólise enzimática. Estudos seguintes realizados in vivo, aplicando a ileostomia, confirmaram a presença de ARs no intestino grosso com possível fermentação local. Deste modo, a partir de 1992, a definição de AR passou a ser mais relacionada aos seus efeitos biológicos, sendo a soma do amido e produtos de sua degradação não absorvida no intestino delgado de indivíduos saudáveis e, portanto, não fornecedora de glicose ao organismo (Nugent, 2005). Na análise química dos alimentos, mesmo apresentando características químicas, organolépticas e alguns efeitos fisiológicos distintos da fibra alimentar, geralmente os ARs são quantificados junto com essa fração devido a não realização da solubilização com hidróxido de potássio ou dimetilsulfóxido (Jenkins et al., 1998).

$\mathrm{O}$ AR pode ser classificado como sendo de quatro tipos, os quais são AR1, AR2, AR3 (figura 1) ou AR4. O AR1 representa o grânulo de amido fisicamente inacessível na matriz do alimento devido à presença de parede celular e proteínas. Pertencem a este grupo grãos inteiros ou parcialmente moídos provindos dos cereais e leguminosas (Lobo e Silva, 2003; Walter et al., 2005). O AR2 refere-se aos grânulos de amido nativos que apresentam lenta digestibilidade devido às características intrínsecas da estrutura dos seus grânulos. É o único que mantém a estrutura e resistência mesmo durante o processo e preparação de muitos alimentos
(Fontinha e Correia, 2010). Encontra-se este tipo em batatas cruas, bananas verdes e amidos com alto teor de amilose. O AR3 consiste em polímeros de amido retrogradado, principalmente amilose, produzidos quando o amido é resfriado após a gelatinização (Lobo e Silva, 2003). Alguns exemplos de AR3 são as batatas e flocos de milho submetidos a altas temperaturas, seguidas de um arrefecimento. O AR4 descreve um grupo de amido modificado quimicamente através de ésteres, fosfatos e éteres, assim como amidos com ligações cruzadas, de tal forma que diminui sua digestibilidade no intestino delgado (Fontinha e Correia, 2010).

Existem diversos fatores que podem influenciar a formação do AR, tais como: cristalinidade do amido, estrutura granular, proporção amilose:amilopectina, retrogradação da amilose, comprimento da cadeia da amilose, linearização da amilopectina, interação do amido a outros componentes, condições de processamento e condições de armazenamento. O AR, independente do tipo, tem um tamanho de partícula pequena, aparência branca, sabor suave, baixa capacidade de retenção de água e viscosidade. As propriedades físicas do AR, particularmente sua capacidade de retenção de água, permite ser um ingrediente funcional que oferece melhor crocância, expansão e textura do alimento pronto para cães (Sajilata et al., 2006).

Por possuir consideráveis teores de AR2, podendo chegar até $84 \%$, o amido da banana verde tem sido bastante estudado na área de nutrição (Uehara, 2011). Uma importante alternativa para o incremento na cadeia produtiva da banana seria a produção de farinhas com qualidades funcionais, o que incentivaria o uso industrial e minimizaria as perdas pós-colheita (Ramos et al., 2009). As farinhas de banana podem ser obtidas por secagem natural ou artificial de bananas verdes ou

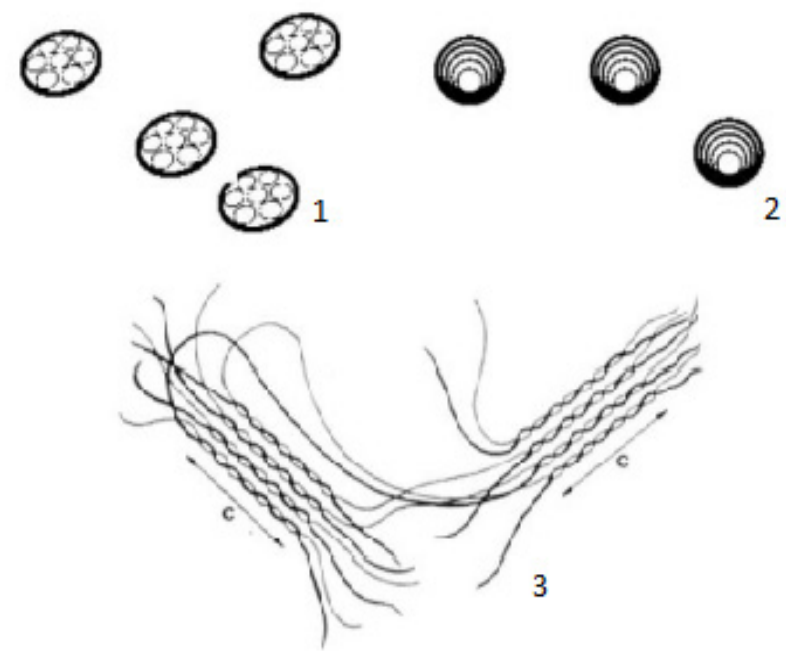

Figura 1. 1. Visão microscópica da estrutura do amido resistente tipo $1 ; 2$. Visăo microscópica da estrutura do amido resistente tipo 2; 3. Apresentação esquemática do amido resistente tipo 3; c. Hélices duplas são ordenadas em uma estrutura cristalina (1.Microscopic view of type 1 resistant starch structure; 2 . Microscopic view of type 2 resistant starch structure; 3 . Schematic presentation of type 3 resistant starch; c. Double helices are ordered in a crystal structure). Fonte: adaptado de Sajilata et al. (2006). 
parcialmente verdes das variedades Prata, Terra, Cavendish, Nanica ou Nanicão. Sua qualidade depende de vários fatores, tais como matéria-prima, modo de secagem, técnicas de procedimento e forma de armazenamento (Uehara, 2011). Além das fontes naturais, e da obtenção via extrusão, também estão disponíveis muitos produtos comerciais de AR, como por exemplo, Novelose $330 \AA$, Pinefiber $\AA$, Fibersol $\AA$, Hi-maize ${ }^{\circledR}$ e CrystaLean $\AA$. As formas de tratamento, fibra alimentar e níveis de AR de alguns produtos sintéticos comerciais estão descritos na tabela I. Quase todos os processos patenteados são baseados na tendência do amido rico em amilose, à retrogradação, ou formas ricas em regiões cristalinas que são resistentes à hidrólise enzimática (Crosby, 2001). Amido de milho rico em amilose tem altas temperaturas de gelatinização, requerendo temperaturas que não são alcançadas em processamentos comuns $\left(154\right.$ a $\left.171^{\circ} \mathrm{C}\right)$ antes dos grânulos serem completamente rompidos. O primeiro AR comercial foi o Hi-Maize® em 1993, originalmente obtido a partir de um híbrido de milho cultivado na Austrália, o qual continha cerca de $80-85 \%$ de amilose com aproximadamente $30 \%$ de AR (Brown et al., 1995). Este amido de milho de alta amilose, classificado com AR2 era vendido em todo mundo e usado em produtos como barras de cereais, cereais, biscoitos, outros produtos de panificação e laticínios. Existem no mercado produtos contendo 47 a $60 \%$ de AR2, como Novelose $\AA 240$ e Novelose ${ }^{\circledR} 260$. A Novelose ${ }^{\circledR} 240$ é um AR2 termicamente modificado baseado em híbrido de milho, o que torna o grânulo nativo mais estável mantendo o amido a altas temperaturas $\left(60\right.$ a $\left.160^{\circ} \mathrm{C}\right)$ na presença limitada de água (10-80\%. Novelose ${ }^{\circledR} 260$ contém $60 \%$ de AR2, o mais alto nível de AR disponível. A Novelose 330® e CrystaLean ${ }^{\circledR}$ também derivam do amido de milho rico em amilose, entretanto, são exemplos comerciais de AR3. O produto CrystaLean ${ }^{\circledR}$ é produzido primeiramente pela hidratação total e ruptura dos grânulos de amido, seguido por um processo enzimático desramificador. Então a mistura é tratada através de ciclos térmicos para atingir um nível elevado de retrogradação antes da secagem, resultando no produto final com aproximadamente $41 \%$ de AR (Sajilata et al., 2006).

Quanto à fonte a ser utilizada em alimentos para cães, há uma série de vantagens no uso de fontes comerciais sintéticas de AR em produtos alimentares, uma vez que ao contrário de fontes naturais, a citar legumes, batatas e bananas, os ARs comerciais não são afetados pelo processamento e condições de armaze- namento (Nugent, 2005). Por exemplo, a quantidade de AR2 na banana verde diminui com o aumento maturação, no entanto, uma forma comercial de AR2, não experimenta essa dificuldade. A tolerância ao tipos de processamento deve ser considerada aoglico selecionar e utilizar o AR para uma determinada aplicação. A maioria dos ARs comerciais mantem suas características através de processos típicos de panificação e de extrusão leve, entretanto, condições extremas de processamento podem danificar o AR (Sajilata et al., 2006).

\section{AproveltAMENTO dO AMIDO DIETÉTICO E OS EFEITOS FISIOLÓGICOS DO AMIDO RESISTENTE}

Os cães apresentam certa particularidade, comparados aos humanos, quanto à ação enzimática sobre os carboidratos, pois não possuem $\alpha$-amilase salivar. No intestino delgado, as ligações $\alpha-1,4$, tanto da amilose quanto da amilopectina, são rompidas pela a $\alpha$-amilase originada do pâncreas. A $\alpha$-amilase não cliva as unidades isoladas de glicose, e sim a cada duas ou três moléculas de glicose fornecendo como produto final maltose ou maltotriose, respectivamente. Em seguida, essas moléculas são lisadas pela maltase, enzima específica da fase de membrana, produzindo duas e três moléculas de glicose, respectivamente, as quais serão absorvidas. O processo digestivo da amilopectina ocorre da mesma forma, exceto para as ligações $\alpha-1,6$ nos pontos de ramificação, as quais não são hidrolisadas. Uma vez que esses pontos de ramificação não são hidrolisados, formam-se oligossacarídeos de cadeia ramificada chamados de dextrina-limite, bem como um dissacarídeo com ligação $\alpha-1,6$ conhecido como isomaltose (Cunningham, 2004). Nos alimentos, as moléculas de amiloses encontram-se reunidas em um arranjo granular que dificulta entrada e saída de água interior deste. Com a menor infiltração de água no interior do grânulo, o acesso das enzimas digestivas torna-se difícil, possibilitando o acesso das amilases basicamente às extremidades, resultando na liberação mais lenta de glicose, porém com maior constância e durante um maior período de tempo quando comparado aos grânulos de amilopectina (Lara, 2005).

Até a década de 80, acreditava-se que o amido era completamente hidrolisado, sendo absorvido no intestino delgado na forma de glicose. No entanto, fatores como relação amilose:amilopectina, forma física do alimento e inibidores enzimáticos podem influenciar a

Tabela I. Procedimento de tratamento, fibra alimentar e os níveis de amido resistente (AR) das diferentes fontes sintéticas comerciais de AR (Treatment procedure, dietary fiber and resistant starch (RS) levels of the different commercial synthetic sources of RS.

\begin{tabular}{lccccc}
\hline & Hylon VII & Hi-maize 1043 & Hi-maize 240 & Hi-maize 260 & Novelose330 \\
\hline Tratamento & Nenhum & Hidrotermal & Hidrotermal & Hidrotermal & Retrogradação \\
Tipo de AR & AR2 & AR2 & AR2 & AR2 & AR3 \\
Quantidade de AR(\%) & 48 & 50 & 53 & 46 & 47 \\
Fibra dietética total (\%) & 18 & 62 & 40 & 60 & 36 \\
\hline
\end{tabular}

Fonte: adaptado de Le Leu et al., 2009). 
taxa de hidrólise do amido e absorção de glicose. Desse modo, o amido pode escapar à digestão no intestino delgado, sendo fermentado no cólon (Wolf et al., 1999), gerando assim diversos benefícios fisiológicos ao animal, como quando ocorre no consumo do AR.

Os ARs interferem em diversos fatores fisiológicos e/ou metabólicos, tais como: melhora na resposta glicêmica, insulinêmica e no perfil lipídico, redução no valor calórico dos alimentos, aumento da saciedade e absorção de micronutrientes, além de agir como prebiótico estimulando o crescimento e atividade de bactérias benéficas colônicas, mantendo consequentemente a saúde do trato gastrintestinal (Fontinha e Correia, 2010; Nugent, 2005). Quando o AR é comparado com fibras solúveis e insolúveis, observa-se que o AR é fisiologicamente analisado como uma fibra solúvel e quimicamente como uma fibra insolúvel, entretanto proporciona melhor desempenho (tabela II) (Pereira, 2007).

\section{DIGESTIBILIDADE DE NUTRIENTES}

A presença de AR na alimentação animal parece interferir positiva ou negativamente na digestibilidade dos nutrientes conforme o elemento estudado. Quando se trata da influencia do AR sobre o aproveitamento dos minerais, são encontrados vários estudos em ratos. Estudando o AR em tal espécie, Lopez et al. (2001) examinaram o efeito de duas fontes de AR2, as quais foram o amido de batata cru (510 g de amido de trigo/100 g da dieta +200 g de amido de batata crú /100 $\mathrm{g}$ da dieta) e amido de milho rico em amilose (510 $\mathrm{g}$ de amido de trigo/100 g da dieta $+200 \mathrm{~g}$ de amido de milho rico em amilose/100 g da dieta), comparadas à uma dieta controle (710 g de amido de trigo/100 g da dieta. Os autores encontraram aumento na biodisponibilidade de cálcio, magnésio, zinco, ferro e cobre nos animais alimentados com AR. Tais resultados coincidem com os encontrados por Schulz et al. (1993), também avaliando em ratos. Os autores observaram os efeitos do amido de milho nativo e retrogradado sobre a absorção de cálcio, magnésio e fósforo. As dietas foram: sem AR, com baixo teor de AR (2 g/100 g da dieta na matéria seca), com AR2 (24,7 g/100 g da dieta na matéria seca) e com AR3 (17,1 g/100 g da dieta na matéria seca. Houve maior absorção de cálcio e magnésio em animais alimentados com dieta contendo AR2, e menor absorção de fósforo nos animais alimentados com dietas contendo AR3. Heijnen et al. (1996) ao alimentarem ratos com dietas contendo AR3 (116 g de AR/ kg da dieta), AR2 (116 g/kg da dieta), glicose, ou lactulose (90,5 g de lactulose/ $\mathrm{kg}$ da dieta) notaram que a absorção aparente de magnésio foi maior no grupo AR2 do que no grupo AR3, entretanto, a digestibilidade verdadeira foi semelhante entre esses tratamentos. Sobre o aproveitamento dos demais nutrientes, Walter et al. (2005) pesquisaram a influência de diferentes teores de AR (0\%, 3\%, 9\% e $18 \%$ de AR Novelose 260® em substituição do amido de milho) em dietas para ratos. Os níveis de 9 e $18 \%$ de AR promoveram o aumento da excreção de nitrogênio nas fezes, bem como redução na digestibilidade aparente da matéria seca.

Em cães, Murray et al. (1998) ao compararem dietas enterais controle (ricas em carboidrato não estrutural), contendo V-complex (complexo lipídio-amilopectina) ou AR (amido retrogradado de milho rico em amilose em substituição ao carboidrato presente na dieta controle) em animais ileostomizados observaram menor digestibilidade da matéria seca, matéria orgânica, proteína bruta e carboidrato no trato gastrintestinal total nos animais que consumiram AR. Mitsuhashi et al. (2010) notaram que as cadelas obesas não castradas no grupo que ingeriram uma dieta com AR apresentaram menor digestibilidade de carboidratos, proteínas, matéria seca e de energia metabolizável em comparação às cadelas dos grupos de dietas com amido de alto índice glicêmico. De maneira semelhante, um estudo recente (Peixoto et al., 2017) encontraram redução da digestibilidade da proteína bruta e gordura para cães idosos que ingeriram dieta com alto AR. Os autores argumentam que o aumento da fermentação no cólon dos cães alimentados com alto AR resultou em maior excreção nitrogênio microbiano via fecal, o que interferiu a digestibilidade aparente da proteína. A digestibilidade da gordura foi justificada pela menor exposição

Tabela II. Propriedades nutricionais de amido resistente e fibras solúvel e insolúvel (Nutritional properties of resistant starch and soluble and insoluble fibers.

\begin{tabular}{|c|c|c|c|}
\hline Propriedades nutricionais & Amido resistente & Fibra solúvel & Fibra insolúvel \\
\hline Insolúvel em água & + & - & + \\
\hline Fermentabilidade & +++ & +++ & - \\
\hline Produção de AGCC & +++ & +++ & - \\
\hline Aumento da produção de butirato & +++ & ++ & - \\
\hline Redução de pH fecal & +++ & +++ & - \\
\hline Aumento da umidade fecal & ++ & ++ & + \\
\hline Aumento da massa fecal (seca) & +++ & + & +++ \\
\hline Redução do tempo de trânsito fecal & ++ & - & +++ \\
\hline
\end{tabular}

Fonte: adaptado de Pereira (2007). 
da célula às enzimas digestivas, no entanto, não foi encontrada pelos autores uma possível explicação de como a ingestão do AR produziu tal efeito.

A redução da digestibilidade dos nutrientes causada pelo AR precisa ser mais bem estudada, contudo pode ser um ponto de restrição de seu uso em dietas não terapêuticas, especialmente para as fases em que há maior demanda nutricional como, por exemplo, crescimento e reprodução.

Produção de ÁCIDOS GRAXOS de CADEIA CURTA E MICROBIOTA INTESTINAL

O AR no cólon é utilizado como substrato para fermentação por bactérias intestinais, sendo os produtos desta fermentação os ácidos graxos de cadeia curta (AGCC) como o acético, o propiônico e o butírico, e gases como hidrogênio, dióxido de carbono e metano. Os principais AGCC são absorvidos a taxas semelhantes em várias regiões do intestino grosso, sendo metabolizados rapidamente pelos colonócitos agindo por fim como importante fonte energética e trófica para as células intestinais. Para Pereira (2007) esses AGCC previnem doenças inflamatórias e podem ajudar a conter o aparecimento de células cancerígenas no cólon. Dos três tipos de AGCC, o butirato é a principal fonte energética intestinal mesmo quando estão disponíveis outros substratos tais como glicose e glutamina; ainda assim, o butirato é aparentemente mais oxidado no cólon proximal do que no distal (Topping e Clifton, 2001).

Estudos em suínos e ratos têm reportado que alimentação com AR aumenta a produção fecal e cecal de AGCC total e também de concentrações individuais de propionato, butirato e acetato. Lopez et al. (2001) avaliando o efeito de duas fontes de AR2, as quais foram o amido de batata cru (510 g de amido de trigo/100 g da dieta $+200 \mathrm{~g}$ de amido de batata cru/100 g da dieta) e amido de milho rico em amilose (510 g de amido de trigo/100 g da dieta $+200 \mathrm{~g}$ de amido de milho rico em amilose/100 g da dieta), comparando a uma dieta controle (710 g de amido de trigo/100 g da dieta) sobre a produção de AGCC em ratos observaram aumento significativo na produção desses ácidos, principalmente propiônico e butírico nos animais que consumiram ambos os tipos de AR. Le Leu et al. (2009) alimentaram ratos com dietas contendo amido de milho com alta amilose rico em AR2 (Hylon $®$ VII, Hi-maize ${ }^{\circledR} 240$, Himaize ${ }^{\circledR} 260$ ) e AR3 (Novelose $\left.® 330\right)$, todos incluídos ao nível de $200 \mathrm{~g} / \mathrm{kg}$ da dieta. Foi possível observar que o $\mathrm{pH}$ fecal e cecal foram menores em todos os grupos alimentados com AR quando comparado ao grupo controle e aquele alimentado com celulose. As dietas com AR aumentaram significativamente AGCC. Os AGCC são relativamente fracos com $\mathrm{pK}$ aproximadamente de 4,8 e se elevadas suas concentrações são capazes de diminuir o pH da digesta, sendo a fonte dietética importante para a intensidade desse efeito. Menores valores de $\mathrm{pH}$ através do aumento de AGCC parecem evitar a proliferação de bactérias patogênicas sensíveis ao pH (Topping e Clifton, 2001).

Além disto, os AGCC podem interferir no fluxo sanguíneo colônico (Mortensen et al., 1991). Em cães, a infusão retal de acetato e propionato aumentaram em 18,1 e 3,1\%, respectivamente, o fluxo sanguíneo colônico, mas foi reduzido em 3,4\% quando o butirato foi infundido. Os mecanismos de ação podem envolver redes neurais locais, bem como quimiorreceptores, juntamente com efeitos diretos sobre as células musculares lisas (Kvietys e Granger, 1981). O aumento do fluxo sanguíneo promovido pelo acetato e propionanto é importante para o efeito proliferativo dos colonócitos. Diferentemente dos resultados encontrados em ratos (Le Leu et al., 2009) e humanos (Topping e Clifton, 2001), Beloshapka et al. (2014) não notaram diferenças na produção de AGCC ao estudarem a ingestão de até $5 \mathrm{~g}$ de AR/dia em cães adultos. Por outro lado, Peixoto et al. (2017) avaliaram dietas com baixo e elevado AR $(0,21$ e $1,46 \%$, respectivamente) ingeridas por cães idosos. Os pesquisadores não obtiveram diferenças entre as dietas sobre os parâmetros histológicos da mucosa intestinal. Porém, ressaltaram que a ingestão da dieta com elevado AR aumentou a produção de propionato, butirato, ácidos graxos voláteis totais e lactato, com consequente redução do $\mathrm{pH}$ fecal. Uma explicação entre a discordância dos resultados dos trabalhos em cães citados acima pode ser devido às distintas fases fisiológicas estudadas. Sabe-se que na fase idosa há um declínio natural da microbiota intestinal benéfica (Larsen e Farcas, 2014) a qual pode ser beneficiada com a ingestão de AR, tornando o seu efeito mais evidente, no entanto, tal situação pode não ser verdadeira nos animais adultos e saudáveis. Neste contexto, não foram encontrados trabalhos que abordassem o assunto para as demais etapas fisiológicas do cão.

Os prebióticos são ingredientes alimentares não digeríveis que estimulam o crescimento e/ou atividade de bactérias potencialmente benéficas do cólon, melhorando a saúde do hospedeiro (Topping et al., 2003) e contribui para aumentar o aproveitamento da dieta com redução da excreção de nitrogênio ao ambiente (Rodrigues et al., 2013). Probiótico refere-se a culturas de microrganismos vivos os quais quando fornecidos aos animais podem melhorar as propriedades da microbiota colônica. E os simbióticos correspondem a uma mistura de pre e probióticos, onde há uma interação sinérgica entre um probiótico específico e um prebiótico particular (Topping et al., 2003). O AR parece funcionar como um prebiótico e simbiótico (Brown et al., 1997; Wang et al., 1999). Tem sido revelado em suínos e ratos que o consumo de dietas com alto conteúdo de AR levou a uma mudança tempo-dependente em AGCC fecal e no intestino grosso, sugerindo uma mudança na população microbiana autóctone. No estudo em suínos alimentados com AR foi observado maior número fecal de bifidobactérias $(8,91 \log 10$ $\mathrm{ufc} / \mathrm{g}$ ) quando comparado a animais alimentados com 
amido de baixa amilose (8,12 log10 ufc/g) (Brown et al., 1997). O AR pode agir como substrato alimentar para Bifidobactéria in vitro. Estudo in vitro mostrou que AR tipo 2 e 4 podem se associar fisicamente com diversas espécies de bifidobactérias protegendo-as de ataque durante a preparação e armazenamento dos alimentos, bem como durante o trânsito gastrintestinal (Wang et al., 1999). Além desses efeitos prebióticos, o AR também parece exercer outras ações promotoras de saúde intestinal. A suplementação com AR associado à terapia de hidratação oral reduziu a perda de líquido e o tempo de recuperação de pessoas com diarréia provocada pela cólera (Ramakrishna et al., 2000). Similares benefícios foram encontrados em crianças, com outras formas de diarréia infecciosa após alimentação com banana verde, e em suínos infectados com Brachyspira yodo dysenteriae alimentados com arroz cozido (Hampson et al., 2000). Acredita-se que o AR pode conferir esses benefícios através do aumento da absorção de líquido como resultado da maior absorção de AGCC. Os AGCC estimulam a captação de água e cátions (sódio, potássio e cálcio) no cólon proximal e, através da sua ação sobre a atividade muscular e no fluxo sanguíneo no cólon reduzindo diretamente a gravidade da diarreia (Topping et al., 2003). Neste sentido, o AR pode ser um importante aliado na terapia de cães convalescentes.

\section{EXCREÇÃO E CONSISTÊNCIA FECAL}

As características fecais são importantes para avaliar a saúde de um cão. Há uma carência de estudos testando os benefícios potenciais do AR no manejo da constipação, porém, devido aos efeitos benéficos da suplementação com AR sobre o volume e consistência fecal e tempo de trânsito, é possível que o aumento da ingestão de AR ajude a melhorar estas condições.

Em ratos, Walter et al. (2005) averiguaram a influência de diferentes teores de AR ( $0 \%, 3 \%, 9 \%$ e 18\% de AR Novelose 260® em substituição do amido de milho) em dietas e observaram que os maiores níveis aumentaram produção fecal. Além disso, é importante considerar quando se trata da qualidade fecal, além da quantidade o tipo de AR. Schulz et al. (1993) estudaram dietas sem AR, com baixo teor de AR (2 g/100 g da dieta na matéria seca), com AR2 (24,7 g/100 g da dieta na matéria seca) e com AR3 (17, 1 g/100 g da dieta na matéria seca) ressaltaram diferença significativa entre os tratamentos quanto à produção fecal com base na matéria seca, de modo que o grupo que consumiu AR3 apresentou maior produção fecal, seguido dos animais alimentados com dieta contendo AR2. De modo semelhante, Le Leu et al. (2009) estudaram dietas contendo amido de milho com alta amilose rico em AR2 (Hylon® VII, Hi-maize ${ }^{\circledR} 240$, Hi-maize ${ }^{\circledR 260)}$ ) e AR3 (Novelose ${ }^{\circledR 330), ~ t o d o s ~ i n c l u i ́ d o s ~ n o ~ n i ́ v e l ~ d e ~} 200$ $\mathrm{g} / \mathrm{kg}$ da dieta, visualizaramque o maior consumo da dieta e excreção fecal ocorreu no grupo de animais na dieta da Novelose $₫ 330$ em relação aos demais grupos alimentados com AR. Em estudos com humanos, o consumo de 30 a 39 g por dia de AR, além de aumentar a produção de fezes, facilitou a defecação, embora tenha provocado aumento da flatulência (Phillips et al., 1995; Jenkins et al., 1998).

Já em cães ileostomizados, Murray et al. (1998) acharam maiores peso (úmido e seco) e o teor de matéria seca das fezes no grupo de animais que consumiu AR ao avaliarem dietas enterais controle (rica em carboidrato não estrutural), contendo V-complex (complexo lipídio-amilopectina) ou AR (retrogradado amido de milho rico em amilose em substituição ao carboidrato presente na dieta controle). Fezes mais bem formadas também foram encontradas neste mesmo grupo. Já Goudez et al. (2011) testaram diferentes fontes e níveis de AR sobre a qualidade fecal de cães de diferentes raças e portes. A dieta de manutenção dos cães foi suplementada em quantidades crescentes de AR de duas fontes: amido de milho com alta amilose (2,5, 4,3 e 7,4\% de AR) e fécula de batata crua $(7,4$ e 11,4\%) de AR. Os cães de pequeno porte mostraram pouca sensibilidade ao AR com base no escore fecal, enquanto que cães de grande porte foram bastante sensíveis à sua suplementação e passaram a ter fezes mais macias. Acredita-se que a sensibilidade de cães de grande porte para AR pode ser ligada, em parte, a maior atividade fermentativa no cólon. Este estudo ressalta que se a fisiologia e eficiência digestiva podem variar dependendo do tamanho ou raça, os níveis de AR também pode ser diferente entre cães. Mais estudos são necessários para entender melhor este fenômeno.

\section{IMPLICAÇÕES METABÓLICAS DO AMIDO RESISTENTE}

\section{METABOLISMO DOS CARBOIDRATOS}

O índice glicêmico (IG) é referido como um recurso auxiliar no controle da glicemia pós-prandial, o que é de fundamental importância em indivíduos com alterações no metabolismo glicídico (Uehara, 2011). Ele está intimamente ligado ao termo resposta glicêmica, e ambos referem-se à capacidade de um determinado alimento em elevar as concentrações de glicose pós-prandial. Desse modo, alimentos com alto índice glicêmico liberam glicose rapidamente na corrente sanguínea, enquanto os alimentos com baixo índice glicêmico liberam glicose mais lentamente na corrente sanguínea, resultando na melhor resposta glicêmica e insulinêmica. Estes ainda podem modular a oxidação de gordura (Nugent, 2005). As diferenças nas respostas glicêmicas e insulinêmica ao amido da dieta estão diretamente relacionadas à taxa de digestão do amido (Uehara, 2011). Recentemente tem havido um grande interesse público e comercial no conceito de índice glicêmico e sua possível inclusão nos rótulos de alimentos com o objetivo de auxiliar no manejo da diabetes e para indicar alimentos potenciais que podem ajudar na perda e manejo de peso. Sabe-se que a fibra dietética pode contribuir para uma melhor resposta glicêmica e 
em geral, alimentos ricos em fibra são atribuídos a um menor índice glicêmico.

Assim como as fibras, o AR também parece contribuir para a queda do índice glicêmico dos alimentos, proporcionando uma menor resposta glicêmica e consequentemente menor resposta insulínica, o que o torna importante no tratamento de diabetes. Entretanto, conforme observado por Caruso e Menezes (2000), flocos de milho produziram elevada resposta glicêmica mesmo contendo elevado teor de AR3 e amido rapidamente digerido. Esse fato pode decorrer do alto percentual de área amorfa, a qual absorve água mais rapidamente, tornando-a mais susceptível à hidrólise enzimática (Luz et al., 1997). Nos grânulos com alto teor de amilopectina, a digestão é mais rápida devido às ramificações, contribuindo assim no aumento da superfície exposta à hidrólise enzimática. Já no alimento rico em amilose, a resposta glicêmica pode ser menor devido à formação de complexos entre esta molécula e ácidos orgânicos, lipídeos e fatores antinutricionais (Caruso e Menezes, 2000).

Raben et al. (1994) objetivaram avaliar o efeito do AR sobre as concentrações plasmáticas de glicose pós-prandial em humanos alimentados com 50 gramas de amido pré-gelatinizado ( $0 \%$ de AR) ou com 50 gramas de amido de batata cru (54\% de AR) e observaram que as concentrações plasmáticas de glicose e de insulina pós-prandial foram significativamente menores após o consumo da refeição contendo altos teores de AR.

Em cães obesos, Kimura (2013) estudou os efeitos inibidores do AR sobre a resposta glicêmica pós-prandial e observou as alterações nas concentrações de glicose no sangue e no IG após a administração de amidos resistentes contidos em soluções de dextrina não digerível (DND) e em $\beta$-dextrina cíclica ( $\beta$-DC) comparados à uma solução de amido solúvel (AS). Como resultado observou redução rápida da concentração de glicose à valores iniciais nos cães que ingeriram DND e as concentrações permaneceram inalteradas para os ingeriram $\beta$-DC quando comparados aos que ingeriram a solução de AS. Além disso, os níveis de IG foram menores nos animais que ingeriram soluções contendo $\mathrm{AR}$ àqueles que receberam a solução de AS. Com esses resultados, o autor evidencia a importância do AR em tratamento dietético de diabetes e obesidade em cães. Já Ribeiro (2016) avaliando os efeitos de dietas com baixo e elevado AR (0,21 e 1,46\%, respectivamente) em cães adultos e idosos sobre as respostas pós-prandiais de glicose e insulina, observou que apenas nos idosos o maior consumo de AR apresentou efetividade em reduzir a glicemia com aumento da secreção de insulina. Portanto, o AR pode ser um importante aliado dietético no controle da diabetes nos cães nas situações em que são mais predispostos à doença, como na fase idosa e/ ou com sobrepeso.

\section{METABOLISMO DOS LIPÍDIOS}

Em ratos, foi observado que o AR parece afetar o metabolismo lipídico levando à diminuição de até 22-32\% nos níveis de colesterol plasmático e 29-42\% de triglicerídeos plasmáticos (Nugent, 2005). A inda em ratos, Lopez et al. (2001) avaliando duas fontes de AR2, as quais foram o amido de batata cru (510 g de amido de trigo/100 g da dieta $+200 \mathrm{~g}$ de amido de batata crú/100 g da dieta) e amido de milho rico em amilose (510 g de amido de trigo/100 g da dieta +200 $\mathrm{g}$ de amido de milho rico em amilose/100 g da dieta), comparando a uma dieta controle (710 g de amido de trigo/100 g da dieta) sobre o metabolismo lipídico observaram redução na absorção de colesterol em ambos os grupos que consumiram AR. O AR também foi eficaz na redução do colesterol e triglicérides plasmáticos. Não houve efeito das dietas sobre colesterol em HDL, entretanto as fontes de AR reduziram o colesterol em lipoproteínas (especialmente lipoproteínas ricas em triglicerídeos) no fígado dos animais alimentados com ambos os ARs. De Deckere et al. (1993) ao avaliarem dieta com baixa $(0,8 \mathrm{~g} / \mathrm{MJ})$ e alta $(9,6 \mathrm{~g} / \mathrm{MJ})$ quantidade de AR observaram redução nas concentrações de colesterol total sérico após uma semana de experimento e nas concentrações de triacilgliceróis sérico após duas semanas de experimento.

Igualmente aos resultados obtidos em ratos, Mitsuhashi et al. (2010) observaram que cadelas obesas não castradas ao ingerirem uma dieta com AR (17 g/100 g de amido de milho, após o processo de extrusão) e diacil glicerol durante 8 semanas apresentaram maior redução do peso corporal e redução da concentração do colesterol total sérico comparadas àquelas que ingeriram dietas com amido de alto índice glicêmico (sem AR). Deste modo, o AR demonstrou potencial como ferramenta na gestão de peso em cães.

\section{CONSIDERAÇÕES FINAIS}

Assim como observado em outras espécies pode-se inferir, por meio da revisão de literatura realizada, que o AR é um interessante alimento para cães, sobretudo em dietas terapêuticas como nos casos de obesidade, diabetes ou animais com distúrbios digestivos. $\mathrm{Na}$ fase idosa, aonde há declínio natural da capacidade fermentativa, a ingestão de AR pode contribuir para o aumento da produção dos AGVs.

A aplicação do AR em alimentos funcionais para cães, além desses benefícios fisiológicos, também pode ser justificada pelo potencial tecnológico devido a fácil inclusão, seja em dietas caseiras ou industrializadas, e pela interferência positiva na expansão, textura e crocrância dos alimentos mais tradicionalmente utilizados para cães como o extrusado completo seco. No entanto, falta o melhor entendimento de como uma dose especifica, o tipo e a origem do AR podem influenciar na resposta desejada nas diferentes etapas fisiológicas bem como os efeitos frente aos diversos portes físicos da espécie canina. 


\section{AGRADECIMENTOS}

Os autores agradecem ao Conselho Nacional de Desenvolvimento Científico e Tecnológico (CNPq) pela concessão de bolsas de estudos.

\section{BIBLIOGRAFÍA}

Beloshapka, A.N.; Alexander, G.L.; Buff, P.R. and Swanson, K.S. 2014 The effects of feeding resistant starch on apparent total tract macronutrient digestibility, faecal characteristics and faecal fermentative end-products in healthy adult dogs. J Nutr Sci, 3: 1-5.

Biörck, I.; Granfeldt, Y.; Lilieberg, H.; Tovar, J. and Asp, N.G. 1994. Food properties affecting the digestion and absorption of carbohydrates. Am Soc Clin Nutrn, 59: 699S-705S.

Brown, I.L.; McNaught, K.J. and Moloney, E. 1995. Hi-maize ${ }^{\mathrm{TM}}$ : new directions in starch technology and nutrition. Food Aust, 47: 272-275.

Brown, I.L.; Warhurst, M.; Arcort, J.; Playne, M.; Illman, R.J. and Topping, D.L. 1997. Fecal numbers of bifidobacteria are high in pigs fed Bifidobacterium longum with a high amylose (amylomaize) starch than with a low amylomaize starch. J Nutr, 9: 1822-1827.

Caruso, L. and Menezes, E.W. 2000. Índice glicêmico dos alimentos. Nutrire, 19/20: 49-64

Crane, S.; Griffin, R. and Messent, P. 2000. Introduction to commercial pet foods. In: R.L. Remillard and P. Roudebush (Eds.). Small Anim Clin Nutr (4 ed.). Mark Morris Institute. Topeka. Kansas.

Crosby, G.A. 2001. Commercial processes for the manufacture of resistant starch. AACC Annual Meeting. Oct.14-2001. Opta Food Ingredients, Inc. Charlotte, N.C. pp. 14-18

Cunningham, J.G. 2004. Digestão e absorção: os processos não fermentativos. In: J.G. Cunningham (Ed.). Tratado de Fisiologia Veterinária (3 ed.). Guanabara Koogan. Rio de Janeiro. pp. 268-269.

De Deckere, E.A.; Kloots, W.J. and van Amelsvoort, J.M. 1993. Resistant starch decreases serum total cholesterol and triacylglycerol concentrations in rats. J Nutr, 123: 2142-2151.

Denardin, C.C. and da Silva, L.P. 2009. Estrutura dos grânulos de amido e sua relação com propriedades físico-químicas. Ciên Rural, 39: 945-954

Dziezak, D.J. 1989. Single- and twin-screw extruders in food processing. Food Technol, 44: 164-174.

Englyst, H.N.; Kingman, S.M. and Cummings, J.H. 1992. Classification and measurement of nutritionally important starch fractions. Eur J Clinl Nutr, 46 (Suppl 2): S33-S50.

Englyst, H.; Wiggins, H.S. and Cummings, J.H. 1982. Determination of the non-starch polysaccharides in plant foods by gas-liquid chromatography of constituent sugars as alditol acetates. Analyst, 107: 307-318.

Fontinha, C. and Correia, P. 2010. Amido resistente em diversas fontes não convencionais de amido. Millenium, 38: 67-81.

Goudez, R.; Weber, M.; Biourge, V. and Nguyen, P. 2011. Influence of different levels and sources of resistant starch on faecal quality of dogs of various. British J Nutr, 106 (Suppl 1): S21 1-S215.

Hampson, D.J.; Robertson, I.D.; La, T.; Oxberry, S.L. and Pethick, D.W. 2000. Influences of diet and vaccination on colonisation of pigs by the intestinal spirochaete Brachyspira (Serpulina) pilosicoli. Vet Microbiol, 73: 75-84.

Heijnen, M.L.; van den Berg, G.J. and Beynen, A.C. 1996. Dietary raw versus retrograded resistant starch enhances apparent but not true magnesium absorption in rats. J Nutr, 126: 2253-2259.

Huang, D.P. 2001 . Selecting an optimum starch for snack development. Cereal Foods Worls, 46: 237-239.

Jenkins, D.J.; Vuksan, V.; Kendall, C.W.; Würsch, P.; Jeffcoat, R.; Waring, S.; Mehling, C.C; Vidgen, E.; Augustin, L.S. and Wong, E. 1998. Physiological effects of resistant starches on fecal bulk, short chain fatty acids, blood lipids and glycemic index. J Am Coll Nutr, 17: 609-616.

Kimura, T. 2013. The regulatory effects of resistant starch on glycaemic response in obese dogs. Arch Anim Nutr, 67: 503-509.
Kleessen, B.; Stoof, G.; Proll, J.; Schmiedl, D.; Noack, J. and Blaut, M. 1997. Feeding resistant starch affects fecal and cecal microflora and short-chain fatty acids in rats. J Anim Sci, 75: 2453-2462.

Kvietys, P.R. and Granger, D.N. 1981. Effect of volatile fatty acids on blood flow and oxygen uptake by the dog colon. Gastroenterology, 80: 962-969.

Lankhorst, C.; Tran, Q.D.; Havenaar, R.; Hendriks, W.H. and van der Poel, A.F. 2007. The effect of extrusion on the nutritional value of canine diets as assessed by in vitro indicators. Anim Feed Sci Technol, 138: 285-297.

Lara, L.B. 2005. Características físico-químicas do amido em alimentos comerciais para cães e gatos. II Simpósio de nutrição e alimentação de cães e gatos. Anais. Lavras. pp. 71-80.

Larsen, J.A. and Farcas, A. 2014. Nutrition of aging dogs. Vet Clin North Am. Small Anim Pract, 44: 741-759.

Le Leu, R.K.; Hu, Y.; Brown, I.L. and Young, G.P. 2009. Effect of high amylose maize starches on colonic fermentation and apoptotic response to DNA-damage in the colon of rats. Nutr Metab (March): 6-11.

Lobo, A.R. and Silva, G.M. 2003. Amido resistente e suas propriedades físico-químicas. Rev Nutr, 16: 219-226.

Lopez, H.W.; Levrat-Verny, M.A.; Coudray, C.; Besson, C.; Krespine, V.; Messager, A.; Demigné, C. and Rémésy, C. 2001. Class 2 resistant starches lower plasma and liver lipids and improve mineral retention in rats. J Nutr, 131: 1283-1289.

Luz, S.S.; Campos, P.L.; Ribeiro, S.M. and Tirapegui, J. 1997. Aspecto atual da digestão e absorção de carboidratos. Arqu Gastroenterol, 34: 175-185.

Mitsuhashi, Y.; Nagaoka, D.; Ishioka, K.; Bigley, K.E.; Okawa, M.; Otsuji, K. and Baver, J.E. 2010. Postprandial lipid-related metabolites are altered in dogs fed dietary diacylglycerol and low glycemic index starch during weight loss. J Nutr, 140, 1815-1823.

Mortensen, F.V.; Hessov, I.; Birke, H.; Korsgaard, N. and Nielsen, H. 1991. Microcirculatory and trophic effects of short chain fatty acids in the human rectum after Hartmann's procedure. BrJ Surg, 78: 1208-1211.

Murray, S.M.; Patil, A.R.; Fahey Jr, G.C.; Merchen, N.R.; Wolf, B.W.; Lai, C. and Garleb, K.A. 1998. Apparent digestibility of a debranched amylopectin-lipid complex and resistant starch incorporated into enteral formulas fed to ileal-cannulated dogs. J Nutr, 128: 2032-2035.

Nugent, A.P. 2005. Health properties of resistant starch. Nutr Bull / BNF, 30: 27-54.

Parker, R. and Ring, S.G. 2001. Aspects of the physical chemistry of starch. J Cereal Sci, 34: 1-17.

Peixoto, M.C.; Ribeiro, É.M.; Maria, A.P.; Loureiro, B.A.; di Santo, L.G.; Putarov, T.C.; Yoshitoshi, F.N.; Pereira, G.T.; Sá, L.R.M. and Carciofi, A.C. 2017. Effect of resistant starch on the intestinal health of old dogs: fermentation products and histological features of the intestinal mucosa. J Anim Physiol Anim Nutr, 26. doi: 10.1111/ipn.12711.

Pereira, K.D. 2007. Amido resistente, a última geração no controle de energia e digestão saudável. Ciênc Tecnol Aliment, 27 (Supl. 1): 88-92.

Phillips, J.; Muir, J.G.; Birkett, A.; Lu, Z.X.; Jones, G.P.; O’Dea, K. and Young, G.P. 1995. Effect of resistant starch on fecal bulk and fermentation-dependent events in humans. Am J Clin Nutr, 62: 121-130.

Raben, A.; Tagliabue, A.; Christensen, N.J.; Madsen, J.; Holst, J J. and Astrup, A. 1994. Resistant starch: the effect on postprandial glycemia, hormonal response, and satiety. Am J Clin Nutr, 60: 544-551.

Ramakrishna, B.S.; Venkataraman, S.; Srinivasan, P.; Dash, P.; Young, G.P. and Blinder, H.J. 2000. Amylase-resistant starch plus oral rehydration solution for cholera. N Engl J Med, 342: 308-313.

Ramos, D.P.; Leonal, M. and Leonel, S. 2009. Amido resistente em farinhas de banana verde. Alimentos e Nutrição, 20: 479-483.

Ribeiro, É.M. 2016. Amido resistente, digestibilidade e respostas pósprandiais de glicose e insulina em cães adultos e idosos. Dissertação (Mestrado). Faculdade de Ciências Agrárias e Veterinárias. Universidade Estadual Estadual Paulista. Jaboticabal. Brasil.

Rodrigues, M.; Pozza, P.C.; Pozza, M.S.; Possamai, M.; Bruno, L.D.; Richart, E.; Wochner, M.O. and Pereira Júnior, J.M. 2013. Effects of 
inulin and a probiotic mixture on nutrient digestibility and nitrogen balance in piglets. Arch Zootec, 62: 255-264.

Sajilata, M.G.; Singhal, R.S. and Kulkarni, P.R. 2006. Resistant starch-A review. Comp Rev Food Sci Food Safety, 5: 1-17.

Schulz, A.G.; van Amelsvoort, J.M. and Beynen, A.C. 1993. Dietary native resistant starch but not retrograded resistant starch raises magnesium and calcium absorption in rats. J Nutr, 123: 1724-1731.

Singh, N.; Singh, J.; Kaur, L.; Sodhi, N.S. and Gill, B.S. 2003. Morphological, thermal and rheological properties of starches from different botanical sources. Food Chem, 81: 219-231.

Spears, J. K. and Fahey Junior, G.C. 2004. Resistant starch as related to companion animal nutrition. J AOAC Inter, 87: 787-791.

Tester, R.F.; Karkalas, J. and Qi, X. 2004. Starch - composition, fine structure and architecture. J Cereal Sci, 39: 151-165.

Topping, D.L. and Clifton, P.M. 2001. Short-chain fatty acids and human colonic function: roles of resistant starch and nonstarch polysaccharides. Physiol Rev, 81: 1031-1064

Topping, D.L.; Fukushima, M. and Bird, A.R. 2003. Resistant starch as a prebiotic and synbiotic: state of the art. Proc Nutr Soc, 62: 171-176.
Tran, Q.D.; Hendriks, W.H. and van der Poel, A.F. 2008. Effects of extrusion processing on nutrients in dry pet food. J Sci Food Agric, 88: 1487-1493.

Uehara, V.B. 2011 . Efeito da radiação gama em propriedades da farinha de banana verde. (Mestrado). Dissertação. Instituto de Pesquisas Energéticas e Nucleares. Universidade do Estado de São Paulo. São Paulo. Walter, M.; da Silva, L.P. and Emanuelli, T. 2005. Amido resistente: características físico-químicas, propriedades fisiológicas. Ciênc Rural, 35: 974-980

Wang, X.; Conway, P.L.; Brown, I.L. and Evans, A.J. 1999. In vitro utilization of amylopectin and high-amylose maize (Amylomaize) starch granules by human colonic bacteria. Appl Environ Microbiol, 65: 4848-4854

Wolf, B.W.; Bauer, L.L. and Fahey Junior, G.C. 1999. Effects of chemical modification on in vitro rate and extent of food starch digestion: an attempt to discover a slowly digested starch. J Agric Food Chem, 47: $4178-4183$ 